\title{
Printable nanomedicines: the future of customized drug delivery?
}

\author{
Maren Preis ${ }^{1} \&$ Jessica M Rosenholm ${ }^{* 1}$ \\ 'Pharmaceutical Sciences Laboratory, Faculty of Science \& Engineering, Åbo Akademi University, BioCity (3rd floor), Tykistökatu 6A, FI - 20521 \\ Turku, Finland \\ *Author for correspondence: jerosenh@abo.fi89002017
}

First draft submitted: 11 April 2017; Accepted for publication: 28 April 2017; Published online: 21 August 2017

Keywords: drug delivery • nanomedicine • nanoparticles • printing

The introduction of printing technologies to emerge medicine and other pharmaceuticals includes printing as alternative fabrication method for dosage forms and drug delivery systems [1] and cell-based constructions for diagnostics, implants and even for potential organ replacements [2]. The advantages of printing technologies for multiple applications lay at hand; printing enables flexible, fast and individual solutions for multiple applications. It is not only possible to print liquid materials onto surfaces (2D), but also to build $3 \mathrm{D}$ constructs.

A printing system using active pharmaceutical ingredients (APIs) opens up opportunities to fabricate tailored and customized drug delivery systems for individual patients. Although marketed products are yet to be established, more personalized medicinal products are beneficial in particular for special patient groups. These include for instance the elderly, who often face multimorbidity and administration issues; and also others, such as children [3]. Since the majority of active pharmaceutical ingredients and, especially new chemical entities (up to $90 \%$ ) have poor solubility, the research community in this area is very interested in overcoming solubility issues by using new technologies. Combining an inkjet printing and drug nanonization procedure has been described in recent literature, [4] and furthermore, the use of nanosuspensions as inks has been successfully demonstrated [5,6]. In these cases, the API itself has been formulated as a nanosuspension, but certain advantages could be expected by incorporating the API into a nanocarrier that is subsequently formulated into an ink, but this is still a relatively unexplored area.

It becomes obvious that the application of printing technologies for medicine requires the development of high standard suitable materials, which endure not only the process itself, but also help keeping the active ingredients stable. Here, nanotechnology, that is, using nanoparticle systems for either diagnostics or delivery appears promising in this regard. Nanoparticles have been widely explored as drug delivery systems mainly aimed for parenteral administration in suspension form, and some of the recognized advantages could be well exploited for printed formulations. Encapsulation of active ingredients into nanocarriers is aimed toward enhancing the stability and solubility of the drug, protecting the drug from the environment (harsh $\mathrm{pH}$ in the GI tract, metabolic enzymes, etc.), promoting sufficient retention of the API within the carrier throughout the delivery process and finally providing sustained, controlled or even stimuli-responsive release, preferentially at the target site. Crossing of biological barriers and cellular uptake as well as controlled intracellular trafficking and release can also be readily provided by the nanosystem, if relevant for the intended application. Consequently, accurate dosing of highly potent, very poorly soluble drugs or fragile biomolecules would gain obvious benefits from being formulated as nano-inks (i.e., API encapsulated in a nanocarrier).

The mentioned advantages could be both process and application related. For instance, nanoparticles have an optimal size to be processed in high-precision printing processes. Printheads used for inkjet printing processes are known for having very small nozzle diameters. The droplet size starts from a few picoliters. Therefore, suspensions are usually more challenging to print. By using a nanoparticular system as drug carrier and simultaneous stabilizer in the ink, the printability can be facilitated. Inkjet printing processes are regulated thermally or using a piezo-electric element to jet the droplets. Printers designed for bioprinting are usually pressure regulated. 
However, even though the heat exposure in a thermal inkjet process can be considered negligible for most substances due the short exposure time, for more sensitive substances, such as biomolecules, the incorporation into a nanocarrier can ensure protection from heat during print processing. Furthermore, unstable APIs could acquire long-term stability when stored as encapsulated in nanoparticles as a nano-ink both before and after printing. Especially inorganic nanoparticles are heat insensitive and can provide good thermal protection. If controlled release is required, the release mechanism could be built into the nanosystem, for example, by utilization of different coatings [7] while immediate release could be achieved for poorly soluble drugs by incorporation into, for example, porous nanoparticles, due to stabilization in amorphous form in the constrained space [8].

Printing processes based on hot-melt extrusion (additive manufacturing process) where the material is exposed to very high temperatures to achieve a polymer melt are not suitable for every type of biomaterial, but for instance, some enzymes are known to have high temperature stability. In combination with functional nanoparticles, the particles cannot only serve as carriers for active substances, but also serve as material property influencing agents, for example, rigidity. The same course of action should prevent recrystallization of the printed drugs over time, as opposed to when the API were printed in free form. Once a nano-ink has been optimized for printing, the drug molecule could also be interchanged more flexibly than if the ink would have to be reformulated for each API, since the nanoparticle platform could, in the best case, be more or less generic.

In the 3D construction of biofunctional scaffolds, nanocarriers can be introduced for monitoring and control of cell behavior, for example, tracking of stem cells, guiding of stem cell transplantation and obtaining controlled and targeted delivery of stem cell signals [9]. The research and development in this field is only starting off, but a first glimpse at recent publications reveals the potential of combining the functionality of nanoparticular matrices and systems and printing technologies in 2D and 3D.

Recent examples include inkjet printing of cyclodextrin-complexed paclitaxel for overcoming its poor solubility and release control of the hydrophilic drug cidofovir by encapsulation into polycaprolactone nanoparticles [10]. On the 3D printing side, drug-eluting implants have been prepared using polylactic acid and the biocompatible ceramic material hydroxyapatite-based nanoparticles as matrix [11]. Zhu et al. [12] used a 3D printer and a unique $3 \mathrm{D}$ printed nano-ink consisting of hydroxyapatite nanoparticles suspended in a hydrogel to create a biomimetic bone-specific environment for evaluating breast cancer bone invasion, which could potentially serve as tool for studying metastasis and assessing drug sensitivity. Mesoporous bioactive glass and mesoporous silica nanoparticles have been used to build a 3D implantable antitubercular composite scaffold to be used to fill surgical defects after surgical treatment of osteoarticular tuberculosis, where the well-defined porous structure of the nanoparticles material was beneficial for the drug loading and release profiles [13]. A fluorescent nanoparticle ink can be used for a 3D quick response code serving as drug anticounterfeiting strategy [14] and colored nanoparticles could, also, be utilized for identification purposes. Nanoparticles used for biomedical research are generally 'colored', in other words, frequently fluorescently tagged by necessity in that they can be detected within physiological/biological environments using biomedical imaging techniques. Unless they are inherently detectable, which is the case for many inorganic nanoparticles [15], there are a range of established methods for rendering these visible whereby also the incorporated (most often fluorescent) dyes obtain similar benefits as nanoencapsulated APIs, such as photostabilization compared with free dye [16].

The potential for nanomaterials to create highly sophisticated drug delivery or diagnostic systems becomes evident from the gained increasing interest during recent years. Current research shows that accurate and personalized dosing is more relevant than ever. The awareness of the society for personalized medicine to meet the individual needs of patients lead to new concepts and approaches to bring the preparations of medicine closer to the patient by means of flexible printing processes. The use of nanoparticular systems in combination with this flexible manufacturing process will potentially evolve during the next years, leading to a range of printable nanomedicines.

\footnotetext{
Financial \& competing interests disclosure

The authors have no relevant affiliations or financial involvement with any organization or entity with a financial interest in or financial conflict with the subject matter or materials discussed in the manuscript. This includes employment, consultancies, honoraria, stock ownership or options, expert testimony, grants or patents received or pending, or royalties.

No writing assistance was utilized in the production of this manuscript.
} 


\section{References}

1 Sandler N, Preis M. Printed drug-delivery systems for improved patient treatment. Trends Pharmacol. Sci. 37, 1070-1080 (2017).

2 Hong N, Yang GH, Lee J et al. 3D bioprinting and its in vivo applications. J. Biomed. Mater. Res. B Appl. Biomater. doi:10.1002/ jbm.b.33826 (2017) (Epub ahead of print).

3 Preis M, Öblom H. 3D-printed drugs for children - are we ready yet? AAPS PharmSciTech. 18(2), 303-308 (2017).

4 Cheow WS, Kiew TY, Hadinoto K. Combining inkjet printing and amorphous nanonization to prepare personalized dosage forms of poorly-soluble drugs. Eur. J. Pharmaceut. Biopharmaceut. 96, 314-321 (2015).

5 Palo M, Kolakovic R, Laaksonen T et al. Fabrication of drug-loaded edible carrier substrates from nanosuspensions by flexographic printing. Int. J. Pharm. 494(2), 603-610 (2015).

6 Pardeike J, Strohmeier DM, Schrodl N et al. Nanosuspensions as advanced printing ink for accurate dosing of poorly soluble drugs in personalized medicines. Int. J. Pharm. 420(1), 93-100 (2011).

7 Aznar E, Oroval M, Pascual L et al. Gated materials for on-command release of guest molecules. Chem. Rev. 116, 561-718 (2016).

8 Rengarajan GT, Enke D, Steinhart M et al. Stabilization of the amorphous state of pharmaceuticals in nanopores. J. Mater. Chem. 18, 2537-2539 (2008).

9 Rosenholm JM, Zhang J, Lindén M et al. Mesoporous silica nanoparticles in tissue engineering - a perspective. Nanomedicine 11(4), 391-402 (2016).

10 Varan C, Wickström H, Sandler $\mathrm{N}$ et al. Inkjet printing of antiviral PCL nanoparticles and anticancer cyclodextrin inclusion complexes on bioadhesive film for cervical administration. Int. J. Pharmaceut. doi:10.1016/j.ijpharm.2017.04.036 (2017) (Epub ahead of print).

11 Water JJ, Bohr A, Boetker J et al. Three-dimensional printing of drug-eluting implants: preparation of an antimicrobial polylactide feedstock material. J. Pharm. Sci. 104(3), 1099-1107 (2015).

12 Zhu W, Holmes B, Glazer RI et al. 3D printed nanocomposite matrix for the study of breast cancer bone metastasis. Nanomedicine 12(1), 69-79 (2016).

13 Zhu M, Li K, Zhu Y et al. 3D-printed hierarchical scaffold for localized isoniazid/rifampin drug delivery and osteoarticular tuberculosis therapy. Acta Biomater. 16, 145-155 (2015).

14 You M, Lin M, Wang S et al. Three-dimensional quick response code based on inkjet printing of upconversion fluorescent nanoparticles for drug anti-counterfeiting. Nanoscale 8(19), 10096-10104 (2016).

15 Giner-Casares JJ, Henriksen-Lacey M, Coronado-Puchau M et al. Inorganic nanoparticles for biomedicine: where materials scientists meet medical research. Materials Today 19(1), 19-28 (2016).

16 Desai D, Șen Karaman D, Prabhakar N et al. Design considerations for mesoporous silica nanoparticulate systems in facilitating biomedical applications. Mesoporous Biomater. 1, 16-43 (2014). 
